\title{
MIXED IMPEDANCE BOUNDARY VALUE PROBLEMS FOR THE LAPLACE-BELTRAMI EQUATION ${ }^{\dagger}$
}

\author{
L.P. CASTRO, R. DUDUCHAVA AND F.-O. SPECK
}

\begin{abstract}
This work is devoted to the analysis of the mixed impedance-Neumann-Dirichlet boundary value problem (MIND BVP) for the Laplace-Beltrami equation on a compact smooth surface $\mathcal{C}$ with smooth boundary. We prove, using the Lax-Milgram Lemma, that this MIND BVP has a unique solution in the classical weak setting $\mathbb{H}^{1}(\mathcal{C})$ when considering positive constants in the impedance condition. The main purpose is to consider the MIND BVP in a nonclassical setting of the Bessel potential space $\mathbb{H}_{p}^{s}(\mathcal{C})$, for $s>1 / p$, $1<p<\infty$. We apply a quasilocalization technique to the MIND BVP and obtain model Dirichlet-Neumann, Dirichletimpedance and Neumann-impedance BVPs for the Laplacian in the half-plane. The model mixed Dirichlet-Neumann BVP was investigated by R. Duduchava and M. Tsaava (2018). The other two are investigated in the present paper. This allows to write a necessary and sufficient condition for the Fredholmness of the MIND BVP and to indicate a large set of the space parameters $s>1 / p$ and $1<p<\infty$ for which the initial BVP is uniquely solvable in the nonclassical setting. As a consequence, we prove that the MIND BVP has a unique solution in the classical weak setting $\mathbb{H}^{1}(\mathcal{C})$ for arbitrary complex values of the nonzero constant in the impedance condition.
\end{abstract}

1. Formulation of the problem. Let $\mathcal{S} \subset \mathbb{R}^{3}$ be some smooth, closed, orientable surface, bordering a compact inner $\Omega^{+}$and outer $\Omega^{-}:=\mathbb{R}^{3} \backslash \overline{\Omega^{+}}$domains. By $\mathcal{C}$ we denote a subsurface of $\mathcal{S}$, which has two faces $\mathcal{C}^{-}$and $\mathcal{C}^{+}$and inherits the orientation from $\mathcal{S}: \mathcal{C}^{+}$

2010 AMS Mathematics subject classification. Primary 35J57; Secondary 45E10, $47 \mathrm{~B} 35$.

Keywords and phrases. Boundary value problem, Laplace-Beltrami equation, impedance-Neumann-Dirichlet condition, potential method, boundary integral equation, Fredholm criteria, symbol, Fredholm property, unique solvability, Bessel potential space.

†Accepted author's manuscript (AAM) (Castro, Luis; Duduchava, Roland; Speck, Frank-Olme. Mixed impedance boundary value problems for the Laplace-Beltrami equation. J. Integral Equations Applications 32 (2020), no. 3, 275-292. DOI: 10.1216/jie.2020.32.275). The final publication is available at https://projecteuclid.org/euclid.jiea/1600308142. 
borders the inner domain $\Omega^{+}$and $\mathcal{C}^{-}$borders the outer domain $\Omega^{-}$. The subsurface $\mathcal{C}$ has a smooth boundary $\Gamma:=\partial \mathcal{C}$, which is decomposed into three closed, simply connected parts $\Gamma=\Gamma_{D} \cup \Gamma_{N} \cup \Gamma_{I}$, having in common only their endpoints. In particular, the common end point of $\Gamma_{N}$ and $\Gamma_{I}$ will be denoted by $P_{I N}$. A generalization to the case where each part consists of a finite number of smooth nonintersecting arcs would be immediate and is not carried out here for simplicity.

Let $\boldsymbol{\nu}(\omega)=\left(\nu_{1}(\omega), \nu_{2}(\omega), \nu_{3}(\omega)\right)^{\top}, \omega \in \overline{\mathcal{C}}$ be the unit normal vector field on the surface $\mathcal{C}$ and

$$
\partial_{\nu}:=\sum_{j=1}^{3} \nu_{j} \partial_{j}
$$

be the normal derivative. We will consider the surface divergence $\operatorname{div}_{\mathcal{C}}$, the surface gradient $\nabla_{\mathcal{C}}$ and the Laplace-Beltrami operator on $\mathcal{C}$ written in terms of the Günter's tangent derivatives (see $[\mathbf{1 8}, \mathbf{2 2}, \mathbf{2 3}]$ for more details):

$$
\begin{aligned}
& \operatorname{div}_{\mathcal{C}} \boldsymbol{U}=\mathcal{D}_{1} U_{1}+\mathcal{D}_{2} U_{2}+\mathcal{D}_{3} U_{3}, \quad \nabla_{\mathcal{C}} \varphi=\left(\mathcal{D}_{1} \varphi, \mathcal{D}_{2} \varphi, \mathcal{D}_{3} \varphi\right)^{\top}, \\
& \boldsymbol{U}=\left(U_{1}, U_{2}, U_{3}\right)^{\top} \\
& \Delta_{\mathcal{C}}:=\operatorname{div}_{\mathcal{C}} \nabla_{\mathcal{C}}=\mathcal{D}_{1}^{2}+\mathcal{D}_{2}^{2}+\mathcal{D}_{3}^{2}, \quad \mathcal{D}_{j}:=\partial_{j}-\nu_{j} \partial_{\nu}, j=1,2,3 .
\end{aligned}
$$

Moreover, let $\boldsymbol{\nu}_{\Gamma}(t)=\left(\nu_{\Gamma, 1}(t), \nu_{\Gamma, 2}(t), \nu_{\Gamma, 3}(t)\right)^{\top}, t \in \Gamma$, be the unit normal vector field on the boundary $\Gamma$, which is tangential to the surface $\mathcal{C}$ and directed outside of the surface. Let, finally,

$$
\partial_{\nu_{\Gamma}}:=\sum_{j=1}^{3} \nu_{\Gamma, j} \mathcal{D}_{j}
$$

denote the corresponding normal derivative on the boundary $\Gamma$.

We will study the mixed impedance-Neumann-Dirichlet boundary value problem (MIND BVP) for the Laplace-Beltrami equation in different space settings

$$
\begin{cases}\Delta_{\mathcal{C}} u(t)=f(t), & t \in \mathcal{C}, \\ u^{+}(\tau)=g(\tau), & \tau \in \Gamma_{D}, \\ \left(\partial_{\boldsymbol{\nu}_{\Gamma}} u\right)^{+}(\tau)=h(\tau), & \tau \in \Gamma_{N}, \\ \left(\partial_{\boldsymbol{\nu}_{\Gamma}} u\right)^{+}(\tau)+c u^{+}(\tau)=\kappa(\tau), & \tau \in \Gamma_{I},\end{cases}
$$


where $u^{+}$and $\left(\partial_{\boldsymbol{\nu}_{\Gamma}} u\right)^{+}$denote respectively the Dirichlet and the Neumann traces on the boundary and $c$ is a constant (the impedance coefficient).

Throughout the paper we will consider $c \neq 0$ since otherwise our problem would be simply the Dirichlet-Neumann boundary value problem already analysed in [23, Theorem 14], in [27, § 5.1] in the classical Hilbert space setting (see (3.1) below) and in [19] in the nonclassical Lebesgue space setting (see (3.3) below).

A rigorous analysis of the solvability of the above and similar problems with Dirichlet, Neumann, mixed and impedance boundary condition for the Helmholtz and other elliptic equations is very helpful for understanding the properties of solutions to elliptic boundary value problems in conical domains (see $[\mathbf{2 8}, \mathbf{3 0}, \mathbf{4 0}]$ ).

In $[\mathbf{2 4}, \mathbf{2 5}]$ the authors suggest another approach to the investigation of the model mixed problem for the Helmholtz equation in twodimensional cones by writing an explicit formula for a solution with two different methods. But the setting is classical only (i.e., in Hilbert spaces) and the approach applies only to Dirichlet and Neumann but not to impedance conditions. Other known results are either limited to special situations such as the case of rectangles and depend strongly on the geometry of the domain $[4,5,6,7,9,10,12,13,14,38]$ or rather sophisticated analytical methods are applied $[\mathbf{2 9}, \mathbf{5 0}]$, or precise settings of appropriate function spaces are missing (see, e.g., $[33,47])$. For a historical survey and for further references we recommend $[8,48,50]$.

There exists yet another approach, which can also be applied - the limiting absorption principle. It is based on a variational formulation, the Lax-Milgram Lemma and its generalizations. Such approach is presented, e.g., in $[\mathbf{1}, \mathbf{2}]$. But again, these results are for the classical setting only.

In the 1980s there was suggested to solve canonical diffraction problems in Sobolev spaces, based on the recent development of pseudodifferential equations in domains with corners and, more generally, with a Lipschitz boundary. It was popularized by E. Meister and F.-O. Speck $[13,14,34,35,36,37,43]$, W.L. Wendland [49], A. Ferreira dos Santos $[\mathbf{4 1}]$ and their collaborators in various publications from which we cited just a few. 
In $[15,19]$ a different approach was suggested, which allows investigation of boundary value problems for elliptic partial differential equations in the nonclassical Lebesgue space setting (3.3). The investigation is based on the boundary integral equation method and the solvability results for Mellin convolution equations in the Bessel potential spaces. We apply this approach to the MIND BVP (1.2) and derive its solvability properties in the nonclassical setting. It is worth to note that the derived result is new even for classical Hilbert space case when the constant $c$ in the impedance condition is an arbitrary complex number (see Theorem 1 below).

2. Auxiliary material. Throughout the paper $\mathcal{S}$ denotes a closed, sufficiently smooth, orientable surface in $\mathbb{R}^{n}$ and $\mathcal{C} \subset \mathcal{S}$ denotes a subsurface with a smooth boundary $\Gamma=\partial \mathcal{C}$.

We will work with the Bessel potential spaces $\mathbb{H}_{p}^{s}(\mathcal{S}), \mathbb{H}_{p}^{s}(\mathcal{C}), \widetilde{\mathbb{H}}_{p}^{s}(\mathcal{C})$ and Sobolev-Slobodečkii spaces $\mathbb{W}_{p}^{s}(\mathcal{S}), \mathbb{W}_{p}^{s}(\mathcal{C}), \widetilde{\mathbb{W}}_{p}^{s}(\mathcal{C})$, where $1<p<$ $\infty, s \in \mathbb{R}$. Let us commence with the definition of the Bessel potential space on the Euclidean space $\mathbb{H}_{p}^{s}\left(\mathbb{R}^{n}\right)$, defined as a subset of the space of Schwartz distributions $\mathbb{S}^{\prime}\left(\mathbb{R}^{n}\right)$ endowed with the norm (see [46])

$$
\left\|u\left|\mathbb{H}_{p}^{s}\left(\mathbb{R}^{n}\right)\|:=\|\langle D\rangle^{s} u\right| L_{p}\left(\mathbb{R}^{n}\right)\right\|,
$$

where $\langle D\rangle^{s}:=\mathcal{F}^{-1}\left(1+|\xi|^{2}\right)^{\frac{s}{2}} \cdot \mathcal{F}$ is the Bessel potential operator, and $\mathcal{F}$ and $\mathcal{F}^{-1}$ are the Fourier transformation and its inverse, respectively. For the definition of the Sobolev-Slobodečkii space $\mathbb{W}_{p}^{s}\left(\mathbb{R}^{n}\right)=\mathbb{B}_{p, p}^{s}\left(\mathbb{R}^{n}\right)$ see $[46]$.

The spaces $\mathbb{H}_{p}^{s}(\mathcal{S})$ and $\mathbb{W}_{p}^{s}(\mathcal{S})$ are defined, in general, by a partition of the unity $\left\{\psi_{j}\right\}_{j=1}^{\ell}$ subordinated to some covering $\left\{Y_{j}\right\}_{j=1}^{\ell}$ of $\mathcal{S}$ and local coordinate diffeomorphisms (see $[46,27]$ for details)

$$
\varkappa_{j}: X_{j} \rightarrow Y_{j}, \quad X_{j} \subset \mathbb{R}^{2}, \quad j=1, \ldots, \ell .
$$

The space $\mathbb{W}_{p}^{s}(\mathcal{S})$ coincides with the trace space of $\mathbb{H}_{p}^{s+\frac{1}{p}}\left(\mathbb{R}^{3}\right)$ on $\mathcal{S}$. In the case $p=2$, we shall use the common simpler notation $\mathbb{H}^{s}(\mathcal{S})$ and $\mathbb{W}^{s}(\mathcal{S})$ for the spaces $\mathbb{H}_{2}^{s}(\mathcal{S})$ and $\mathbb{W}_{2}^{s}(\mathcal{S})$, and it is known that $\mathbb{W}^{s}(\mathcal{S})=\mathbb{H}^{s}(\mathcal{S})$ for $s \geq 0,1<p<\infty($ see $[\mathbf{4 6}])$.

For $p=2$, the same simplification in the spaces notation will occur in all the other spaces that we will be using in this paper. 
The space $\widetilde{\mathbb{H}}_{p}^{s}(\mathcal{C})$ is defined as the subspace of $\mathbb{H}_{p}^{s}(\mathcal{S})$ of those functions $\varphi \in \mathbb{H}_{p}^{s}(\mathcal{S})$, which are supported in the closed subsurface (i.e., $\operatorname{supp} \varphi \subset \overline{\mathcal{C}})$, whereas $\mathbb{H}_{p}^{s}(\mathcal{C})$ denotes the quotient space $\mathbb{H}_{p}^{s}(\mathcal{C}):=$ $\mathbb{H}_{p}^{s}(\mathcal{S}) / \widetilde{\mathbb{H}}_{p}^{s}\left(\mathcal{C}^{c}\right)$, and $\mathcal{C}^{c}:=\mathcal{S} \backslash \overline{\mathcal{C}}$ is the complemented subsurface. For $s>1 / p-1$ the space $\mathbb{H}_{p}^{s}(\mathcal{C})$ can be identified with the space of those distributions $\varphi$ on $\mathcal{C}$ which admit extensions $\ell \varphi \in \mathbb{H}_{p}^{s}(\mathcal{S})$, while $\widetilde{\mathbb{H}}_{p}^{s}(\mathcal{C})$ is identified with the space $r_{\mathcal{C}} \mathbb{H}_{p}^{s}(\mathcal{S})$, where $r_{\mathcal{C}}$ is the restriction to the subsurface $\mathcal{C}$ of $\mathcal{S}$.

For $s<0$, those spaces can be defined by duality, e.g., $\mathbb{H}_{p}^{s}(\mathcal{C})=$ $\left(\widetilde{\mathbb{H}}_{q}^{-s}(\mathcal{C})\right)^{\prime}$, where $\frac{1}{p}+\frac{1}{q}=1$. The spaces $\widetilde{\mathbb{W}}_{p}^{s}(\mathcal{C})$ and $\mathbb{W}_{p}^{s}(\mathcal{C})$ are defined similarly.

The Bessel potential $\mathbb{H}_{p}^{s}(\Gamma), \mathbb{H}_{p}^{s}\left(\Gamma_{0}\right), \widetilde{\mathbb{H}}_{p}^{s}\left(\Gamma_{0}\right)$ and Sobolev-Slobodečkii $\mathbb{W}_{p}^{s}(\Gamma), \mathbb{W}_{p}^{s}\left(\Gamma_{0}\right), \widetilde{\mathbb{W}}_{p}^{s}\left(\Gamma_{0}\right)$ spaces on a closed contour $\Gamma$ and an open arc $\Gamma_{0}$ are also defined similarly. In particular, we shall also use $\widetilde{\mathbb{H}}_{p, \Gamma_{1}}^{s}\left(\Gamma_{0}\right)$ and $\widetilde{\mathbb{W}}_{p, \Gamma_{1}}^{s}\left(\Gamma_{0}\right)$ to denote the $\mathbb{H}_{p}^{s}\left(\Gamma_{1}\right)$ and $\mathbb{W}_{p}^{s}\left(\Gamma_{1}\right)$ distributions, respectively, with support on $\Gamma_{0} \subsetneq \Gamma_{1} \subsetneq \Gamma$. This means that, e.g., the distributions of $r_{\Gamma_{0}} \widetilde{\mathbb{H}}_{p, \Gamma_{1}}^{s}\left(\Gamma_{0}\right)$ are extensible by zero to $\Gamma_{1}$ within $\mathbb{H}_{p}^{s}\left(\Gamma_{1}\right)$ (where $r_{\Gamma_{0}}$ denotes the corresponding restriction operator to $\Gamma_{0}$ ).

It is worth noting that for an integer $m=1,2, \ldots$ the Bessel potential $\mathbb{H}_{p}^{m}(\mathcal{S})$ and Sobolev $\mathbb{W}_{p}^{m}(\mathcal{S})$ spaces coincide and an equivalent norm in both spaces may be defined with the help of the Günter's derivatives (see $[\mathbf{1 7}, \mathbf{1 8}, \mathbf{2 2}]$ and see (1.1) for the Günter's derivatives $\left.\mathcal{D}_{1}, \mathcal{D}_{2}, \mathcal{D}_{3}\right)$ :

$$
\left\|u \mid \mathbb{W}_{p}^{m}(\mathcal{S})\right\|:=\left[\sum_{|\alpha| \leqslant m}\left\|\mathcal{D}^{\alpha} u \mid L_{p}(\mathcal{S})\right\|^{p}\right]^{\frac{1}{p}}, \quad \mathcal{D}^{\alpha}:=\mathcal{D}_{1}^{\alpha_{1}} \mathcal{D}_{2}^{\alpha_{2}} \mathcal{D}_{3}^{\alpha_{3}} .
$$

Let us also consider $\widetilde{\mathbb{H}}_{0}^{-1}(\mathcal{C})$, a subspace of $\widetilde{\mathbb{H}}^{-1}(\mathcal{C})$, orthogonal to

$$
\widetilde{\mathbb{H}}_{\Gamma}^{-1}(\mathcal{C}):=\left\{f \in \widetilde{\mathbb{H}}^{-1}(\mathcal{C}):\langle f, \varphi\rangle=0 \text { for all } \varphi \in C_{0}^{1}(\mathcal{C})\right\} .
$$

$\widetilde{\mathbb{H}}_{\Gamma}^{-1}(\mathcal{C})$ consists of those distributions from $\widetilde{\mathbb{H}}^{-1}(\mathcal{C})$ which are supported on $\Gamma$ and $\widetilde{\mathbb{H}}^{-1}(\mathcal{C})$ decomposes into the direct sum of the subspaces:

$$
\widetilde{\mathbb{H}}^{-1}(\mathcal{C})=\widetilde{\mathbb{H}}_{\Gamma}^{-1}(\mathcal{C}) \oplus \widetilde{\mathbb{H}}_{0}^{-1}(\mathcal{C}) .
$$


The space $\widetilde{\mathbb{H}}_{\Gamma}^{-1}(\mathcal{C})$ is nontrivial (see $[\mathbf{2 7}, \S 5.1]$ ) and if the right-hand side $f$ in (1.2) is chosen from the orthogonal subspace, we are able to guarantee the unique solvability of the corresponding BVPs (cf. [27] and $(3.3))$.

3. Main results. For some (sufficiently small) $\epsilon>0$ we shall consider the $\epsilon$-ball $B_{\epsilon}\left(P_{I N}\right)$ centered at $P_{I N}$. Moreover, considering $\Gamma_{I, \epsilon}:=\Gamma_{I} \cap \overline{B_{\epsilon}\left(P_{I N}\right)}$, we will make use of a one-to-one smooth transformation $\vartheta: \Gamma_{I, \epsilon} \rightarrow \Gamma_{N}$ which produces the overlapping of $\Gamma_{I, \epsilon}$ onto $\Gamma_{N, \epsilon^{\vartheta}}:=\vartheta\left(\Gamma_{I, \epsilon}\right) \subset \Gamma_{N}$ and such that $\vartheta\left(P_{I N}\right)=P_{I N}$. Additionally, we will use the notation $\Gamma_{I N, \epsilon}:=\Gamma_{I, \epsilon} \cup \Gamma_{N, \epsilon^{\vartheta}}$ and the map $T_{\vartheta}$ given by $\left(T_{\vartheta} \varphi\right)(x)=\varphi\left(\vartheta^{-1}(x)\right)$, for $x \in \Gamma_{N, \epsilon^{\vartheta}}$.

Theorem 1. The MIND BVP (1.2) has a unique solution in the classical weak setting

$$
\begin{aligned}
& u \in \mathbb{H}^{1}(\mathcal{C}), \quad f \in \widetilde{\mathbb{H}}_{0}^{-1}(\mathcal{C}), \quad g \in \mathbb{H}^{1 / 2}\left(\Gamma_{D}\right), \\
& h \in \mathbb{H}^{-1 / 2}\left(\Gamma_{N}\right), \quad \kappa \in \mathbb{H}^{-1 / 2}\left(\Gamma_{I}\right),
\end{aligned}
$$

for all complex values of the nonzero impedance parameter $c \in \mathbb{C}$ if and only if the following compatibility condition is satisfied:

$$
r_{\Gamma_{N, \epsilon^{\vartheta}}} h-T_{\vartheta}\left(r_{\Gamma_{I, \epsilon}} \kappa\right) \in r_{\Gamma_{N, \epsilon^{\vartheta}}} \widetilde{\mathbb{H}}_{\Gamma_{I N, \epsilon}}^{-1 / 2}\left(\Gamma_{N, \epsilon^{\vartheta}}\right) .
$$

For some very natural and detailed interpretation of the necessity of having compatibility conditions on the data, we refer the reader to $[27,37,42,39,45]$.

The just formulated theorem will be proved first for positive parameters $c>0$ (see Theorem 3), based on the Lax-Milgram Lemma (see Lemma 1 ). Then, the result is extended to arbitrary nonzero complex numbers $c \in \mathbb{C}$ with the help of Theorem 2 (see the proof, concluding Section 7).

From Theorem 1 we cannot even conclude the continuity of a solution, while from the existence of a nonclassical weak solution in the space $\mathbb{H}_{p}^{1}(\mathcal{C})$ for some $2<p<\infty$, we can even prove the Hölder continuity of $u$. It is very important to know the maximal smoothness of a solution as, for example, in designing approximation methods. To this end we will investigate the solvability properties of the MIND BVP 
(1.2) in the nonclassical setting

$u \in \mathbb{H}_{p}^{s}(\mathcal{C}), \quad f \in \widetilde{\mathbb{H}}_{p}^{s-2}(\mathcal{C}) \cap \widetilde{\mathbb{H}}_{0}^{-1}(\mathcal{C}), \quad g \in \mathbb{W}_{p}^{s-1 / p}\left(\Gamma_{D}\right)$, $h \in \mathbb{W}_{p}^{s-1-1 / p}\left(\Gamma_{N}\right), \quad \kappa \in \mathbb{W}_{p}^{s-1-1 / p}\left(\Gamma_{I}\right), \quad 1<p<\infty, \quad s>\frac{1}{p}$,

subjected to the compatibility condition

$$
r_{\Gamma_{N, \epsilon^{\vartheta}}} h-T_{\vartheta}\left(r_{\Gamma_{I, \epsilon}} \kappa\right) \in r_{\Gamma_{N, \epsilon^{\vartheta}}} \widetilde{\mathbb{W}}_{p, \Gamma_{I N, \epsilon}}^{-1 / 2}\left(\Gamma_{N, \epsilon^{\vartheta}}\right),
$$

and find necessary and sufficient conditions of solvability. Note that the constraint $s>1 / p$ is necessary to ensure the existence of the trace $u^{+}$on the boundary (in the sense of the trace theorem [46]).

Besides the practical usefulness of a nonclassical solution to the MIND BVP (1.2), the solvability conditions are much more interesting and far non-trivial, which is seen by comparing Theorem 1 and Theorem 2.

We recall that a bounded linear operator acting between Banach spaces is said to satisfy the Fredholm property (or, in short, is Fredholm) if the dimension of its kernel is finite, the operator has a closed image, and the codimension of the image is also finite. We will say that the MIND BVP (1.2), (3.3)-(3.4) is Fredholm if the operator which characterizes (1.2), (3.3)-(3.4) has the Fredholm property. More precisely, this operator associated with the BVP is defined by $u \mapsto(f, g, h, \kappa)$ as a bounded linear operator in the Banach space setting of (3.3)-(3.4), cf. [42]. Its inverse, if it exists, is known as the resolvent operator.

The following theorem will be proved in the concluding part of Section 7. 


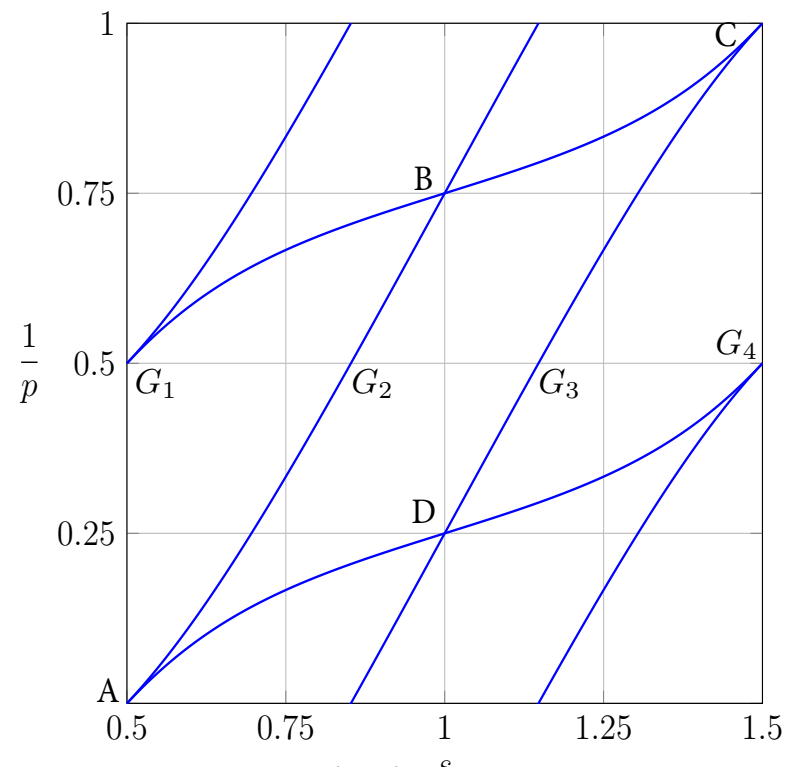

Figure 1. All pairs $\left(s, \frac{1}{p}\right) \stackrel{s}{s}$ of parameters on the curves that violate the condition (3.5).

Theorem 2. Let $1<p<\infty, s>1 / p$. The MIND BVP (1.2) with the compatibility condition (3.4) is Fredholm in the nonclassical setting (see (3.3)) if and only if

$$
\cos ^{2} \pi s-\left|\sin 2 \pi\left(s-\frac{1}{p}\right)\right| \neq 0 .
$$

In particular, the MIND BVP (1.2) has a unique solution $u$ in the nonclassical setting (3.3) if the point $(s, 1 / p)$ belongs to the open curved quadrangle $A B C D$ in Figure 1.

The function in the inequality (3.5) is 1-periodic with respect to the variable $s$ and the condition means that the curves in Figure 1 do not cross the point $(s-k, 1 / p)$, where $k=0,1, \ldots$ is an integer such that $\frac{1}{2}<s-k \leqslant \frac{3}{2}$.

Remark 1. From (3.5) we derive that the MIND BVP (1.2) with (3.4) 
is Fredholm for $p=2$ (see points $G_{1}, G_{2}, G_{3}, G_{4}$ in Figure 1) if:

$$
s \notin\left\{\frac{1}{2}, \frac{3}{2}, \ldots\right\}+\left\{0, \pi-\frac{\arctan 2}{\pi}, \frac{\arctan 2}{\pi}\right\} .
$$

4. Solvability of the MIND BVP in the weak classical setting. In the present section we will prove the unique solvability of the MIND BVP (1.2) in the classical weak setting (3.1)-(3.2), based on the Lax-Milgram Lemma [31, 32].

Lemma 1. (Lax-Milgram) Let $\mathfrak{B}$ be a Banach space, $A(\varphi, \psi)$ be a continuous, bilinear form

$$
A(\cdot, \cdot): \mathfrak{B} \times \mathfrak{B} \rightarrow \mathbb{R}
$$

and positive definite

$$
A(\varphi, \varphi) \geq C\|\varphi \mid \mathfrak{B}\|^{2} \quad \forall \varphi \in \mathfrak{B}, \quad C>0 .
$$

Let $L(\cdot): \mathfrak{B} \rightarrow \mathbb{R}$ be a continuous linear functional.

The linear equation

$$
A(\varphi, \psi)=L(\psi)
$$

has a unique solution $\varphi \in \mathfrak{B}$ for arbitrary $\psi \in \mathfrak{B}$.

We need the following two auxiliary lemmata, which are proved in [17, Lemma 4.8] and in [18], respectively.

Lemma 2. Let $s>0, s \notin \mathbb{N}, 1<p<\infty, \boldsymbol{B}(D)$ be a normal differential operator of the first-order defined in the vicinity of the boundary $\Gamma=\partial \mathcal{C}$ and $\boldsymbol{A}(D)$ be a normal differential operator of the second order defined on the surface $\mathcal{C}$. Then there exists a continuous linear operator

$$
\mathcal{B}: \quad \mathbb{W}_{p}^{s}(\Gamma) \otimes \mathbb{W}_{p}^{s-1}(\Gamma) \longrightarrow \mathbb{H}_{p}^{s+\frac{1}{p}}(\mathcal{C})
$$

such that

$$
(\mathcal{B} \Phi)^{+}=\varphi_{0}, \quad(\boldsymbol{B}(D) \mathcal{B} \Phi)^{+}=\varphi_{1}, \quad \boldsymbol{A}(D) \mathcal{B} \Phi \in \widetilde{\mathbb{H}}_{p}^{s-2+\frac{1}{p}}(\mathcal{C})
$$

for an arbitrary pair of functions $\Phi=\left(\varphi_{0}, \varphi_{1}\right)^{\top}$ where $\varphi_{0} \in \mathbb{W}_{p}^{s}(\Gamma)$ and $\varphi_{1} \in \mathbb{W}_{p}^{s-1}(\Gamma)$. 
Lemma 3. For the Laplace-Beltrami operator $\Delta_{\mathcal{C}}$ on the open surface $\mathcal{C}$ with the boundary $\Gamma=\partial \mathcal{C}$, the following Green formula is valid

(4.6) $\left(\Delta_{\mathcal{C}} v, w\right)_{\mathcal{C}}=\left(\left(\partial_{\nu_{\Gamma}} v\right)^{+}, w^{+}\right)_{\Gamma}-\left(\nabla_{\mathcal{C}} v, \nabla_{\mathcal{C}} w\right)_{\mathcal{C}}, \quad v, w \in \mathbb{W}^{1}(\mathcal{C})$, where $(\varphi, \psi)_{\mathcal{C}}$ denotes the scalar product of vector-functions and

$$
\nabla_{\mathcal{C}} v:=\left(\mathcal{D}_{1} v, \mathcal{D}_{2} v, \mathcal{D}_{3} v\right)^{\top}
$$

denotes the Günter's gradient on the surface $\mathcal{C}$.

Now we can prove the main result of the present section.

Theorem 3. If $c>0$, then the MIND BVP (1.2) has a unique solution in the classical weak setting (3.1)-(3.2).

Proof: We commence by reduction of the BVP (1.2), (3.1)-(3.2) to an equivalent one with the homogeneous Dirichlet condition. For this, we extend the boundary data $g \in \mathbb{W}^{1 / 2}\left(\Gamma_{D}\right)$ up to some function $g_{0} \in \mathbb{W}^{1 / 2}(\Gamma)$ on the entire boundary $\Gamma$ and apply Lemma 2: there exists a function $G \in \mathbb{W}^{1}(\mathcal{C})$ such that $G^{+}=g_{0}$ almost everywhere on the boundary (in particular, $G^{+}(t)=g(t)$ for $t \in \Gamma_{D}$ ) and $\Delta_{\mathcal{C}} G \in \widetilde{\mathbb{W}}^{-1}(\overline{\mathcal{C}})$.

For a new unknown function $v:=u-G$ we have the following equivalent reformulation of the MIND BVP (1.2):

$$
\begin{cases}\Delta_{\mathcal{C}} v(t)=f^{0}(t), & t \in \mathcal{C}, \\ v^{+}(\tau)=0, & \tau \in \Gamma_{D}, \\ \left(\partial_{\boldsymbol{\nu}_{\Gamma}} v\right)^{+}(\tau)=h^{0}(\tau), & \tau \in \Gamma_{N}, \\ \left(\partial_{\boldsymbol{\nu}_{\Gamma}} v\right)^{+}(\tau)+c v^{+}(\tau)=\kappa^{0}(\tau), & \tau \in \Gamma_{I} .\end{cases}
$$

where

$$
\begin{array}{r}
f^{0}:=f-\Delta_{\mathcal{C}} G \in \widetilde{\mathbb{W}}^{-1}(\mathcal{C}), \quad h^{0}:=h-\left(\partial_{\nu_{\Gamma}} G\right)^{+} \in \mathbb{W}^{-1 / 2}\left(\Gamma_{N}\right), \\
\kappa^{0}:=\kappa-\left(\partial_{\nu_{\Gamma}} G\right)^{+}-c G^{+} \in \mathbb{W}^{-1 / 2}\left(\Gamma_{I}\right), \quad v^{+} \in \widetilde{\mathbb{W}}^{1 / 2}\left(\Gamma_{I N}\right),
\end{array}
$$

and

$$
r_{\Gamma_{N, \epsilon}} h_{0}-T_{\vartheta}\left(r_{\Gamma_{I, \epsilon}} \kappa_{0}\right) \in r_{\Gamma_{N, \epsilon \vartheta}} \widetilde{\mathbb{W}}_{\Gamma_{I N, \epsilon}}^{-1 / 2}\left(\Gamma_{N, \epsilon^{\vartheta}}\right) .
$$


To justify the inclusion $v^{+} \in \widetilde{\mathbb{W}}^{1 / 2}\left(\Gamma_{I N}\right)$ note that, due to our construction, the trace of a solution on $\Gamma_{D}$ vanishes $\left.v^{+}\right|_{\Gamma_{D}}=0$.

Note that this reduction of the BVP is a reduction in the sense of [44], i.e., the operators associated to the full BVP and the semihomogeneous BVP, respectively, are equivalent after extension. Consequently, the two BVPs have the same "regularity properties" such as the Fredholm property, invertibility etc. The equivalence after extension relation results from the fact that the extension operator in (4.8) is right invertible, a standard conclusion that makes use of the compatibility condition (3.2). Hence the compatibility condition is sufficient. Its necessity is a consequence of the representation formulas together with the trace theorem. See analogous considerations for boundary-transmission problems for the Helmholtz equation in halfplanes $[42,43,45]$.

Let $\Gamma_{0} \subset \Gamma$ be a part of the boundary and by $\widetilde{\mathbb{W}}^{s}\left(\Gamma_{0}, \mathcal{C}\right), s>1 / 2$, denote the space of functions $\varphi \in \mathbb{W}^{s}(\mathcal{C})$ which have their trace on the boundary supported in $\Gamma_{0}$, i.e., $\varphi^{+} \in \widetilde{\mathbb{W}}^{s-1 / 2}\left(\Gamma_{0}\right)$. Since the traces of functions $\varphi \in \widetilde{\mathbb{W}}^{s}\left(\Gamma_{0}, \mathcal{C}\right)$ vanish on a part of the boundary, the Poincaré inequality

$$
\left\|\varphi\left|\mathbb{L}_{2}(\mathcal{C})\|\leqslant\| \nabla_{\mathcal{C}} \varphi\right| \mathbb{L}_{2}(\mathcal{C})\right\|
$$

holds and, therefore, $\left\|\nabla_{\mathcal{C}} \varphi \mid \mathbb{L}_{2}(\mathcal{C})\right\|$ defines an equivalent norm on this space

$$
\left\|\nabla_{\mathcal{C}} \varphi\left|\mathbb{L}_{2}(\mathcal{C})\|\leqslant\| \varphi\right| \mathbb{W}^{1}(\mathcal{C})\right\| \leqslant C_{1}\left\|\nabla_{\mathcal{C}} \varphi \mid \mathbb{L}_{2}(\mathcal{C})\right\| \quad \forall \varphi \in \widetilde{\mathbb{W}}^{1}\left(\Gamma_{0}, \mathcal{C}\right) .
$$

Let us rewrite the Green formula (4.6) as follows

$$
\begin{aligned}
\left(\nabla_{\mathcal{C}} v, \nabla_{\mathcal{C}} w\right)_{\mathcal{C}}+c\left(v^{+}, w^{+}\right)_{\Gamma_{I}}= & \left(\left(\partial_{\boldsymbol{\nu}_{\Gamma}} v\right)^{+}, w^{+}\right)_{\Gamma_{D}}+\left(\left(\partial_{\boldsymbol{\nu}_{\Gamma}} v\right)^{+}, w^{+}\right)_{\Gamma_{N}} \\
& +\left(\left(\partial_{\boldsymbol{\nu}_{\Gamma}} v\right)^{+}+c v^{+}, w^{+}\right)_{\Gamma_{I}}-\left(\Delta_{\mathcal{C}} v, w\right)_{\mathcal{C}}
\end{aligned}
$$

and insert the data (4.7) from the reformulated boundary value problem (4.8)-(4.9) into it, also take $w=v$ :

$$
\left(\nabla_{\mathcal{C}} v, \nabla_{\mathcal{C}} v\right)_{\mathcal{C}}+c\left(v^{+}, v^{+}\right)_{\Gamma_{I}}=\left(h^{0}, v^{+}\right)_{\Gamma_{N}}+\left(\kappa^{0}, v^{+}\right)_{\Gamma_{I}}-\left(f^{0}, v\right)_{\mathcal{C}} .
$$

The bilinear form in the left-hand side of the equality (4.11) is symmetric and, since $c>0$, also positive definite (at the last step we apply 
the inequality (4.10))

$$
\begin{aligned}
\left(\nabla_{\mathcal{C}} v, \nabla_{\mathcal{C}} v\right)_{\mathcal{C}}+c\left(v^{+}, v^{+}\right)_{\Gamma_{I}} & \geqslant\left\|\nabla_{\mathcal{C}} v \mid \mathbb{L}_{2}(\mathcal{C})\right\|^{2} \\
& \geqslant C_{2}\left\|v \mid \mathbb{W}^{1}(\mathcal{C})\right\|^{2} \quad \forall v \in \widetilde{\mathbb{W}}^{1}\left(\Gamma_{I N}, \mathcal{C}\right) .
\end{aligned}
$$

The functionals $\left(h^{0}, v^{+}\right)_{\Gamma_{N}},\left(\kappa^{0}, v^{+}\right)_{\Gamma_{I}}$ and $\left(f^{0}, v\right)_{\mathcal{C}}$ from the righthand side of the equality (4.11) are correctly defined and continuous, because $h^{0} \in \mathbb{W}^{-1 / 2}\left(\Gamma_{N}\right), \kappa^{0} \in \mathbb{W}^{-1 / 2}\left(\Gamma_{I}\right), f^{0} \in \widetilde{\mathbb{W}}^{-1}(\mathcal{C})$, while their counterparts in the functional belong to the dual spaces $v^{+} \in \widetilde{\mathbb{W}}^{1 / 2}\left(\Gamma_{N}\right)$ and $v \in \widetilde{\mathbb{W}}^{1}\left(\Gamma_{N}, \mathcal{C}\right) \subset \mathbb{W}^{1}(\mathcal{C})$.

By applying the Lax-Milgram Lemma 1 we accomplish the proof.

5. Model mixed BVPs and reduction to boundary pseudodifferential equations. Let us consider three model mixed boundary value problems for the Laplace equation on the upper half-plane $\mathbb{R}_{+}^{2}:=\mathbb{R} \times \mathbb{R}^{+}:$the Dirichlet-Neumann

$$
\begin{cases}\Delta u(x)=f(x), & x \in \mathbb{R}_{+}^{2}, \\ u^{+}(t)=g_{1}(t), & t \in \mathbb{R}^{-}:=(-\infty, 0), \\ -\left(\partial_{2} u\right)^{+}(t)=h_{1}(t), & t \in \mathbb{R}^{+}:=(0, \infty),\end{cases}
$$

the Dirichlet-impedance

$$
\begin{cases}\Delta u(x)=f(x), & x \in \mathbb{R}_{+}^{2}, \\ u^{+}(t)=g_{1}(t), & t \in \mathbb{R}^{-}, \\ -\left(\partial_{2} u\right)^{+}(t)+c u^{+}(t)=\kappa_{1}(t), & t \in \mathbb{R}^{+}\end{cases}
$$

and the Neumann-impedance

$$
\begin{cases}\Delta u(x)=f(x), & x \in \mathbb{R}_{+}^{2}, \\ -\left(\partial_{2} u\right)^{+}(t)=h_{2}(t), & t \in \mathbb{R}^{-}, \\ -\left(\partial_{2} u\right)^{+}(t)+c u^{+}(t)=\kappa_{1}(t), & t \in \mathbb{R}^{+},\end{cases}
$$

boundary value problems. Here $\partial_{\boldsymbol{\nu}_{\Gamma}}=-\partial_{2}$ is the normal derivative on the boundary $\mathbb{R}$ of $\mathbb{R}_{+}^{2}$. 
The BVPs (5.1)-(5.3) will be treated in the nonclassical setting:

$$
\begin{aligned}
g_{1} \in \mathbb{W}_{p}^{s-1 / p}\left(\mathbb{R}^{-}\right), \quad h_{1}, \kappa_{1} & \in \mathbb{W}_{p}^{s-1-1 / p}\left(\mathbb{R}^{+}\right), \quad h_{2} \in \mathbb{W}_{p}^{s-1-1 / p}\left(\mathbb{R}^{-}\right), \\
f & \in \widetilde{\mathbb{H}}_{0}^{-1}\left(\mathbb{R}_{+}^{2}\right), \quad 1<p<\infty, \quad s>\frac{1}{p}
\end{aligned}
$$

and in (5.3) we also have the compatibility condition

$$
h_{2}-J \kappa_{1} \in r_{-} \widetilde{\mathbb{W}}_{p, \mathbb{R}}^{s-1 / p}\left(\mathbb{R}^{-}\right):=r_{-} \widetilde{\mathbb{W}}_{p}^{s-1-1 / p}\left(\mathbb{R}^{-}\right),
$$

where $J$ denotes the reflection operator $J \varphi(x)=\varphi(-x)$.

We say that an operator $A: \mathbb{W}_{p}^{s}(\Omega) \rightarrow \mathbb{W}_{q}^{r}(\Omega), \Omega \subset \mathbb{R}^{n}$, between Besov spaces (or between Bessel potential or, also, between direct products of such spaces) is locally invertible at some point $x_{0} \in \bar{\Omega}:=\Omega \cup \partial \Omega$ if there exists an operator $B_{x_{0}}$ and a smooth function $v_{x_{0}} \in C^{m}(\Omega), m \geqslant \max \{|s|,|q|\}$, with a compact support, which is the identity $v_{x_{0}}(x)=1$ in some neighbourhood $\left|x-x_{0}\right|<\varepsilon$ of $x_{0}$, such that $A B_{x_{0}} v_{x_{0}} I=v_{x_{0}} I, v_{x_{0}} B_{x_{0}} A=v_{x_{0}} I$, where $I$ denotes the identity operator in the appropriate space.

More details about locally invertible operators can be found in [26, Chapter 5] and in [3, Appendix C].

It is clear, that if $A: \mathbb{H}_{p}^{s}(\Omega) \rightarrow \mathbb{H}_{q}^{r}(\Omega)$ is invertible or even Fredholm, then it is locally invertible at all $x_{0} \in \bar{\Omega}$. The inverse statement is obviously false.

Let under invertibility, Fredholmness and local invertibility of boundary value problems (e.g. of (5.1), (5.2) and (5.3)) understand the invertibility, Fredholmness and local invertibility of the corresponding boundary integral operators (of the operators in (5.12), (6.5) and in (6.9), respectively).

Lemma 4. The MIND BVP (1.2) is Fredholm in the nonclassical setting (3.3)-(3.4) if all the three model mixed BVPs Dirichlet-Neumann (5.1), Dirichlet-impedance (5.2) and Neumann-impedance (5.3) are locally invertible at 0 in the nonclassical setting (5.4)-(5.5).

Proof: We refer to [19, Lemma 2.5] for the proof, which applies localization. 
Let us recall the main results about the mixed Dirichlet-Neumann BVP (5.1), obtained in [19].

Consider the Newton, single and double layer potentials for the Laplace equation (see (5.1), (5.2), (5.3))

$$
\begin{aligned}
\boldsymbol{N}_{\mathbb{R}_{+}^{2}} v(x) & :=\frac{1}{4 \pi} \int_{\mathbb{R}_{+}^{2}} \ln \left[\left(x_{1}-y_{1}\right)^{2}+\left(x_{2}-y_{2}\right)^{2}\right] v\left(y_{1}, y_{2}\right) d y_{1} d y_{2} \\
(5.6) \boldsymbol{V}_{\mathbb{R}} v(x) & :=\frac{1}{4 \pi} \int_{\mathbb{R}} \ln \left[\left(x_{1}-\tau\right)^{2}+x_{2}^{2}\right] v(\tau) d \tau, \\
\boldsymbol{W}_{\mathbb{R}} v(x) & :=\frac{x_{2}}{2 \pi} \int_{\mathbb{R}} \frac{v(\tau) d \tau}{\left(x_{1}-\tau\right)^{2}+x_{2}^{2}}, \quad x=\left(x_{1}, x_{2}\right)^{\top} \in \mathbb{R}_{+}^{2},
\end{aligned}
$$

which have the standard boundedness properties

$$
\begin{aligned}
\boldsymbol{N}_{\mathbb{R}_{+}^{2}}: & \mathbb{H}_{p}^{s}\left(\mathbb{R}_{+}^{2}\right) \longrightarrow \mathbb{H}_{p}^{s+2}\left(\mathbb{R}_{+}^{2}\right), \\
\boldsymbol{V}_{\mathbb{R}} & : \quad \mathbb{H}_{p}^{s}(\mathbb{R}) \longrightarrow \mathbb{H}_{p}^{s+1+\frac{1}{p}}\left(\mathbb{R}_{+}^{2}\right), \\
\boldsymbol{W}_{\mathbb{R}} & : \quad \mathbb{H}_{p}^{s}(\mathbb{R}) \longrightarrow \mathbb{H}_{p}^{s+\frac{1}{p}}\left(\mathbb{R}_{+}^{2}\right) .
\end{aligned}
$$

Any solution to one of the model BVP (5.1)-(5.3) in both, the weak classical and nonclassical setting is represented as follows:

$$
\begin{aligned}
(5.7 \mathrm{a}) u(x)= & \boldsymbol{N}_{\mathbb{R}_{+}^{2}} f(x)+\boldsymbol{W}_{\mathbb{R}} u^{+}(x)+\boldsymbol{V}_{\mathbb{R}}\left(\partial_{2} u\right)^{+}(x) \\
= & \boldsymbol{N}_{\mathbb{R}_{+}^{2}} f(x)+\left(\boldsymbol{W}_{\mathbb{R}}+c \boldsymbol{V}_{\mathbb{R}}\right) u^{+}(x) \\
& -\boldsymbol{V}_{\mathbb{R}}\left[-\left(\partial_{2} u\right)^{+}+c u^{+}\right](x) \\
(5.7 \mathrm{~b}) u(x)= & \boldsymbol{N}_{\mathbb{R}_{+}^{2}} f(x)+\left(\frac{1}{c} \boldsymbol{W}_{\mathbb{R}}+\boldsymbol{V}_{\mathbb{R}}\right)\left(\partial_{2} u\right)^{+}(x) \\
& +\frac{1}{c} \boldsymbol{W}_{\mathbb{R}}\left[-\left(\partial_{2} u\right)^{+}+c u^{+}\right](x) \\
(5.7 \mathrm{c}) u(x)= & \boldsymbol{N}_{\mathbb{R}_{+}^{2}} f(x)+(1-c) \boldsymbol{W}_{\mathbb{R}} u^{+}(x)+\left(\boldsymbol{W}_{\mathbb{R}}+\boldsymbol{V}_{\mathbb{R}}\right)\left(\partial_{2} u\right)^{+}(x) \\
& +\boldsymbol{W}_{\mathbb{R}}\left[-\left(\partial_{2} u\right)^{+}+c u^{+}\right](x), \quad u \in \mathbb{W}^{1}\left(\mathbb{R}_{+}^{2}\right), \quad x \in \mathbb{R}_{+}^{2}
\end{aligned}
$$

(see $[\mathbf{2 0}, \mathbf{2 1}, \mathbf{1 9}, \mathbf{1 7}])$. The densities in $(5.7 \mathrm{a})-(5.7 \mathrm{~b})$ represent the Dirichlet $u^{+}$, the Neumann $-\left(\partial_{2} u\right)^{+}$and the impedance $-\left(\partial_{2} u\right)^{+}+c u^{+}$ traces of a solution $u$ on the boundary. 
Let us remind the Plemelji formulas

$$
\begin{aligned}
& \left(\boldsymbol{W}_{\mathbb{R}} \varphi\right)^{ \pm}(t)= \pm \frac{1}{2} \varphi(t), \\
& \left(-\partial_{2} \boldsymbol{V}_{\mathbb{R}} \psi\right)^{ \pm}(t)=\mp \frac{1}{2} \psi(t), \\
& \left(-\partial_{2} \boldsymbol{W}_{\mathbb{R}} \psi\right)^{ \pm}(t)=\boldsymbol{V}_{\mathbb{R},+1} \psi(t), \\
& \left(\boldsymbol{V}_{\mathbb{R}} \varphi\right)^{ \pm}(t)=\boldsymbol{V}_{\mathbb{R},-1} \varphi(t), \quad t \in \mathbb{R}:=\partial \mathbb{R}_{+}^{2},
\end{aligned}
$$

where the pseudodifferential operators

$$
\begin{aligned}
& \boldsymbol{V}_{\mathbb{R},-1} v(t):=\frac{1}{2 \pi} \int_{\mathbb{R}} \ln |t-\tau| v(\tau) d \tau, \\
& \boldsymbol{V}_{\mathbb{R},+1} \varphi(t):=-\frac{1}{2 \pi} \int_{\mathbb{R}} \frac{v(\tau) d \tau}{(t-\tau)^{2}}, \quad t \in \mathbb{R},
\end{aligned}
$$

of orders -1 and +1 , respectively, have the following standard mapping properties (see $[\mathbf{2 0}, \mathbf{2 1}, \mathbf{1 7}, \mathbf{2 7}]$ ):

$$
\begin{aligned}
\boldsymbol{V}_{\mathbb{R},-1} & : \quad \mathbb{H}_{p}^{s}(\mathbb{R}) \longrightarrow \mathbb{H}_{p}^{s+1}(\mathbb{R}), \\
\boldsymbol{V}_{\mathbb{R},+1} & : \quad \mathbb{H}_{p}^{s}(\mathbb{R}) \longrightarrow \mathbb{H}_{p}^{s-1}(\mathbb{R}), \quad s \in \mathbb{R}, \quad 1<p<\infty .
\end{aligned}
$$

Note that the direct values of the double layer potential $W_{\mathbb{R}}$ and its adjoint are zero $W_{\mathbb{R}, 0}=W_{\mathbb{R}, 0}^{*}=0$.

Fix some extensions $g_{0} \in \mathbb{W}_{p}^{s-1 / p}(\mathbb{R})$ and $h_{0} \in \mathbb{W}_{p}^{s-1-1 / p}(\mathbb{R})$ of the boundary data $g_{1} \in \mathbb{W}_{p}^{s-1 / p}\left(\mathbb{R}^{-}\right)$and $h_{1} \in \mathbb{W}_{p}^{s-1-1 / p}\left(\mathbb{R}^{+}\right)$. The difference between such two extensions belong to the spaces

$\varphi_{0}:=u^{+}-g_{0} \in \widetilde{\mathbb{W}}_{p}^{s-1 / p}\left(\mathbb{R}^{+}\right), \quad \psi_{0}:=\left(-\partial_{2} u\right)^{+}-h_{0} \in \widetilde{\mathbb{W}}_{p}^{s-1-1 / p}\left(\mathbb{R}^{-}\right)$,

respectively, and it is sufficient to know the functions $\varphi_{0}$ and $\psi_{0}$ to solve the problem. Indeed, by introducing the boundary values of a solution (5.9) to the BVP (5.1) into the formula (5.7a) we get the representation:

$(5.10) u(x)=\boldsymbol{N}_{\mathbb{R}_{+}^{2}} f(x)+\boldsymbol{W}_{\mathbb{R}}\left(g_{0}+\varphi_{0}\right)(x)-\boldsymbol{V}_{\mathbb{R}}\left(h_{0}+\psi_{0}\right)(x), x \in \mathbb{R}_{+}^{2}$.

To find the unknown functions $\varphi_{0}$ and $\psi_{0}$ in (5.10), in [19] the following system of pseudodifferential equations was derived, which is 
equivalent with the BVP

$$
\begin{gathered}
\left\{\begin{array}{l}
\frac{1}{2} \varphi_{0}+r_{+} \boldsymbol{V}_{\mathbb{R},-1} \psi_{0}=G_{1} \quad \text { on } \quad \mathbb{R}^{+} \\
\frac{1}{2} \psi_{0}-r_{-} \boldsymbol{V}_{\mathbb{R},+1} \varphi_{0}=H_{1} \quad \text { on } \quad \mathbb{R}^{-},
\end{array}\right. \\
\varphi_{0} \in \widetilde{\mathbb{W}}_{p}^{s-1 / p}\left(\mathbb{R}^{+}\right), \quad \psi_{0} \in \widetilde{\mathbb{W}}_{p}^{s-1-1 / p}\left(\mathbb{R}^{-}\right),
\end{gathered}
$$

where

$$
\begin{gathered}
G_{1}:=r_{+}\left[\left(\boldsymbol{N}_{\mathbb{R}_{+}^{2}} f\right)^{+}-\frac{1}{2} g_{0}-\boldsymbol{V}_{\mathbb{R},-1} h_{0}\right] \in \mathbb{W}_{p}^{s-1 / p}\left(\mathbb{R}^{+}\right), \\
H_{1}:=r_{-}\left[\left(-\partial_{2} \boldsymbol{N}_{\mathbb{R}_{+}^{2}} f\right)^{+}-\frac{1}{2} h_{0}+\boldsymbol{V}_{\mathbb{R},+1} g_{0}\right] \in \mathbb{W}_{p}^{s-1-1 / p}\left(\mathbb{R}^{-}\right),
\end{gathered}
$$

and $r_{+}, r_{-}$are the restriction operators from $\mathbb{R}$ to $\mathbb{R}^{+}$and $\mathbb{R}^{-}$, respectively.

The system (5.11) can be written in the operator form

$$
\begin{gathered}
\boldsymbol{A}_{D N}\left(\begin{array}{c}
\varphi_{0} \\
\psi_{0}
\end{array}\right)=\left(\begin{array}{c}
G_{1} \\
H_{1}
\end{array}\right), \quad \boldsymbol{A}_{D N}:=\left(\begin{array}{cc}
\frac{1}{2} r_{+} \boldsymbol{V}_{\mathbb{R},-1} \\
-r_{-} \boldsymbol{V}_{\mathbb{R},+1} & \frac{1}{2} I
\end{array}\right) \\
\boldsymbol{A}_{D N}: \underset{\widetilde{\mathbb{W}}_{p}^{s-1 / p}\left(\mathbb{R}^{+}\right)}{\widetilde{\mathbb{W}}_{p}^{s-1-1 / p}\left(\mathbb{R}^{-}\right)} \rightarrow \underset{\mathbb{W}_{p}^{s-1 / p}\left(\mathbb{R}^{+}\right)}{\mathbb{W}_{p}^{s-1-1 / p}\left(\mathbb{R}^{-}\right)} .
\end{gathered}
$$

6. Investigation of the model boundary pseudodifferential equations. The first result has been proved in [19, Theorem 1.5, Theorem 1.6].

Theorem 4. Let $1<p<\infty, s>1 / p$.

The system of boundary pseudodifferential equations (5.11) is an equivalent reformulation of the mixed Dirichlet-Neumann BVP (5.1) in the setting (5.4)-(5.5).

The operator associated to the system of boundary pseudodifferential equations (5.11) is locally invertible at 0 if and only if the following condition holds

$$
\cos ^{2} \pi s-\left|\sin 2 \pi\left(s-\frac{1}{p}\right)\right| \neq 0 .
$$


Note that the expression "equivalent reformulation" is used here (and in the following) in the very strong sense that the two associated operators are equivalent after extension, see [44] for details.

Now let us consider the mixed Dirichlet-impedance BVP (5.2).

Let $g_{0} \in \mathbb{W}_{p}^{s-1 / p}(\mathbb{R})$ and $\kappa_{0} \in \mathbb{W}_{p}^{s-1-1 / p}(\mathbb{R})$ be arbitrary but fixed extensions of the boundary data $g_{1} \in \mathbb{W}_{p}^{s-1 / p}\left(\mathbb{R}^{-}\right)$and $\kappa_{1} \in$ $\mathbb{W}_{p}^{s-1-1 / p}\left(\mathbb{R}^{+}\right)$. The difference between such two extensions belong to the spaces

$$
\begin{aligned}
\varphi_{0} & :=u^{+}-g_{0} \in \widetilde{\mathbb{W}}_{p}^{s-1 / p}\left(\mathbb{R}^{+}\right), \\
v_{0} & :=\left(-\partial_{2} u\right)^{+}+c u^{+}-\kappa_{0} \in \widetilde{\mathbb{W}}_{p}^{s-1-1 / p}\left(\mathbb{R}^{-}\right)
\end{aligned}
$$

respectively and it is sufficient to know the functions $\varphi_{0}$ and $v_{0}$ to solve the problem. Indeed, by introducing the boundary values $u^{+}$and $\left(-\partial_{2} u\right)^{+}+c u^{+}$from (6.2) into the representation formula (5.7a) we get the representation formula of a solution to the BVP (5.2):

(6.3) $u(x)=\boldsymbol{N}_{\mathbb{R}_{+}^{2}} f(x)+\left(\boldsymbol{W}_{\mathbb{R}}+c \boldsymbol{V}_{\mathbb{R}}\right)\left(g_{0}+\varphi_{0}\right)(x)-\boldsymbol{V}_{\mathbb{R}}\left(\kappa_{0}+v_{0}\right)(x)$, $x \in \mathbb{R}_{+}^{2}$.

By applying the Plemelji formulas (5.8) to (6.3), we get the following:

$$
\left\{\begin{array}{l}
u^{+}=g_{0}+\varphi_{0} \\
\quad=\left(\boldsymbol{N}_{\mathbb{R}_{+}^{2}} f\right)^{+}+\left(\frac{1}{2} I+c \boldsymbol{V}_{\mathbb{R},-1}\right)\left(g_{0}+\varphi_{0}\right)-\boldsymbol{V}_{\mathbb{R},-1}\left(\kappa_{0}+v_{0}\right) \\
-\left(\partial_{2} u\right)^{+}+c u^{+}=\kappa_{0}+v_{0}=-\left(\partial_{2} \boldsymbol{N}_{\mathbb{R}_{+}^{2}} f\right)^{+}+c\left(\boldsymbol{N}_{\mathbb{R}_{+}^{2}} f\right)^{+} \\
+\left(\boldsymbol{V}_{\mathbb{R},+1}+c \boldsymbol{V}_{\mathbb{R},-1}\right)\left(g_{0}+\varphi_{0}\right)+\left(\frac{1}{2} I-c \boldsymbol{V}_{\mathbb{R},-1}\right)\left(\kappa_{0}+v_{0}\right) .
\end{array}\right.
$$

Next we apply the restriction operators $r_{+}$to the first equation and $r_{-}$to the second one and obtain the following system of boundary 
pseudodifferential equations

$$
\begin{gathered}
\text { (6.4) } \begin{array}{c}
\left(\frac{1}{2} I-c r_{+} \boldsymbol{V}_{\mathbb{R},-1}\right) \varphi_{0}+r_{+} \boldsymbol{V}_{\mathbb{R},-1} v_{0}=G_{1} \quad \text { on } \mathbb{R}^{+}, \\
\left(\frac{1}{2} I+c r_{-} \boldsymbol{V}_{\mathbb{R},-1}\right) v_{0}-\left(r_{-} \boldsymbol{V}_{\mathbb{R},+1}+c r_{-} \boldsymbol{V}_{\mathbb{R},-1}\right) \varphi_{0}=K_{1} \text { on } \mathbb{R}^{-},
\end{array} \\
\varphi_{0} \in \widetilde{\mathbb{W}}_{p}^{s-1 / p}\left(\mathbb{R}^{+}\right), \quad v_{0} \in \widetilde{\mathbb{W}}_{p}^{s-1-1 / p}\left(\mathbb{R}^{-}\right), \quad G_{1} \in \mathbb{W}_{p}^{s-1 / p}\left(\mathbb{R}^{+}\right), \\
K_{1}:=r_{-}\left[c\left(\boldsymbol{N}_{\mathbb{R}_{+}^{2}} f\right)^{+}-\left(\partial_{2} \boldsymbol{N}_{\mathbb{R}_{+}^{2}} f\right)^{+}+\left(\boldsymbol{V}_{\mathbb{R},+1}+c \boldsymbol{V}_{\mathbb{R},-1}\right) g_{0}\right. \\
\left.\quad-\left(\frac{1}{2} I+c \boldsymbol{V}_{\mathbb{R},-1}\right) \kappa_{0}\right] \in \mathbb{W}_{p}^{s-1-1 / p}\left(\mathbb{R}^{-}\right) .
\end{gathered}
$$

The system (6.4) can be written in the operator form

$$
\begin{gathered}
\boldsymbol{A}_{D I}\left(\begin{array}{c}
\varphi_{0} \\
v_{0}
\end{array}\right)=\left(\begin{array}{c}
G_{1} \\
K_{1}
\end{array}\right), \\
\boldsymbol{A}_{D I}:=\left(\begin{array}{cc}
\frac{1}{2} I-c r_{+} \boldsymbol{V}_{\mathbb{R},-1} & r_{+} \boldsymbol{V}_{\mathbb{R},-1} \\
-r_{-} \boldsymbol{V}_{\mathbb{R},+1}-c r_{-} \boldsymbol{V}_{\mathbb{R},-1} & \frac{1}{2} I+c r_{-} \boldsymbol{V}_{\mathbb{R},-1}
\end{array}\right) \\
\boldsymbol{A}_{D I}: \underset{\mathbb{W}_{p}^{s-1 / p}\left(\mathbb{R}^{+}\right)}{\mathbb{\mathbb { W }}_{p}^{s-1-1 / p}\left(\mathbb{R}^{-}\right)} \rightarrow \begin{array}{c}
\mathbb{W}_{p}^{s-1 / p}\left(\mathbb{R}^{+}\right) \\
\mathbb{W}_{p}^{s-1-1 / p}\left(\mathbb{R}^{-}\right)
\end{array}
\end{gathered}
$$

Theorem 5. Let $1<p<\infty, s>1 / p$.

The system of boundary pseudodifferential equations (6.4) is an equivalent reformulation of the mixed Dirichlet-impedance BVP (5.2) in the setting (5.4)-(5.5).

The operator associated to the system of boundary pseudodifferential equations (6.4) is locally invertible at 0 if and only if the condition (6.1) holds.

Proof: The operator $\frac{1}{2} I-r_{+} c \boldsymbol{V}_{\mathbb{R},-1}$ is locally equivalent at 0 to $\frac{1}{2} I$, since the second summand has lower order. Similarly, the operator $\frac{1}{2} I+c r_{-} \boldsymbol{V}_{\mathbb{R},-1}$ is locally equivalent at 0 to $\frac{1}{2} I$ and the operator $r_{-} \boldsymbol{V}_{\mathbb{R},+1}+c r_{-} \boldsymbol{V}_{\mathbb{R},-1}$ is locally equivalent at 0 to $r_{-} \boldsymbol{V}_{\mathbb{R},+1}$. Then 
the operators associated to the systems (6.4) and (5.11) are locally equivalent at 0 and Theorem 5 follows from Theorem 4 .

Now let us consider the mixed Neumann-impedance BVP (5.3).

Let $h_{0} \in \mathbb{W}_{p}^{s-1-1 / p}(\mathbb{R})$ and $\kappa_{0} \in \mathbb{W}_{p}^{s-1-1 / p}(\mathbb{R})$ be arbitrary but fixed extensions of the boundary data $h_{2} \in \mathbb{W}_{p}^{s-1-1 / p}\left(\mathbb{R}^{-}\right)$and $\kappa_{1} \in$ $\mathbb{W}_{p}^{s-1-1 / p}\left(\mathbb{R}^{+}\right)$. The difference between such two extensions belong to the spaces

$$
\begin{aligned}
& \psi_{0}:=-\left(\partial_{2} u\right)^{+}-h_{0} \in \widetilde{\mathbb{W}}_{p}^{s-1-1 / p}\left(\mathbb{R}^{+}\right), \\
& v_{0}:=-\left(\partial_{2} u\right)^{+}+c u^{+}-\kappa_{0} \in \widetilde{\mathbb{W}}_{p}^{s-1-1 / p}\left(\mathbb{R}^{-}\right)
\end{aligned}
$$

and it is sufficient to know the functions $\psi_{0}$ and $v_{0}$ to solve the problem. Indeed, by introducing the boundary values $\left(-\partial_{2} u\right)^{+}$and $\left(-\partial_{2} u\right)^{+}+c u^{+}$from (6.2) into the representation formula (5.7b), we get the representation formula of a solution to the BVP (5.3):

$$
\begin{aligned}
u(x)=\boldsymbol{N}_{\mathbb{R}_{+}^{2}} f(x) & -\left(\frac{1}{c} \boldsymbol{W}_{\mathbb{R}}+\boldsymbol{V}_{\mathbb{R}}\right)\left(h_{0}+\psi_{0}\right)(x) \\
& +\frac{1}{c} \boldsymbol{W}_{\mathbb{R}}\left(\kappa_{0}+v_{0}\right)(x), \quad x \in \mathbb{R}_{+}^{2} .
\end{aligned}
$$

By applying the Plemelji formulas (5.8) to (6.7), we get the following:

$$
\left\{\begin{aligned}
-\left(\partial_{2} u\right)^{+} & =h_{0}+\psi_{0}=-\left(\partial_{2} \boldsymbol{N}_{\mathbb{R}_{+}^{2}} f\right)^{+}-\left(\frac{1}{c} \boldsymbol{V}_{\mathbb{R},+1}-\frac{1}{2} I\right)\left(h_{0}+\psi_{0}\right) \\
& +\frac{1}{c} \boldsymbol{V}_{\mathbb{R},+1}\left(\kappa_{0}+v_{0}\right), \\
c u^{+}= & -\left(\partial_{2} u\right)^{+}+c u^{+}+\left(\partial_{2} u\right)^{+}=\kappa_{0}+v_{0}-h_{0}-\psi_{0}=c\left(\boldsymbol{N}_{\mathbb{R}_{+}^{2}} f\right)^{+} \\
& -\left(\frac{1}{2} I+c \boldsymbol{V}_{\mathbb{R},-1}\right)\left(h_{0}+\psi_{0}\right)+\frac{1}{2}\left(\kappa_{0}+v_{0}\right)
\end{aligned}\right.
$$

Thus, we have obtained the following system of boundary pseudodifferential equations

$$
\begin{aligned}
& \left\{\begin{array}{l}
\frac{1}{c} \boldsymbol{V}_{\mathbb{R},+1}\left(\psi_{0}-v_{0}\right)+\frac{1}{2} I \psi_{0}=G, \\
\frac{1}{2} I\left(\psi_{0}-v_{0}\right)-c \boldsymbol{V}_{\mathbb{R},-1} \psi_{0}=K,
\end{array}\right. \\
& \psi_{0} \in \widetilde{\mathbb{W}}_{p}^{s-1-1 / p}\left(\mathbb{R}^{+}\right), \quad v_{0} \in \widetilde{\mathbb{W}}_{p}^{s-1-1 / p}\left(\mathbb{R}^{-}\right),
\end{aligned}
$$




$$
\begin{gathered}
G:=\left(-\partial_{2} \boldsymbol{N}_{\mathbb{R}_{+}^{2}} f\right)^{+}-\frac{1}{2} h_{0}+\frac{1}{c} \boldsymbol{V}_{\mathbb{R},+1}\left(\kappa_{0}-h_{0}\right) \in \mathbb{W}_{p}^{s-2-1 / p}(\mathbb{R}) \\
K:=\frac{1}{2} \kappa_{0}-\frac{1}{2} h_{0}-c\left(\boldsymbol{N}_{\mathbb{R}_{+}^{2}} f\right)^{+}+c \boldsymbol{V}_{\mathbb{R},-1} h_{0} \in \mathbb{W}_{p}^{s-1-1 / p}(\mathbb{R})
\end{gathered}
$$

Theorem 6. Let $1<p<\infty, s>1 / p$.

The system of boundary pseudodifferential equations (6.4) is an equivalent reformulation of the mixed Neumann-impedance BVP (5.3) in the setting (5.4)-(5.5).

The operator associated to the system of boundary pseudodifferential equations (6.4) is locally invertible at 0.

Proof: Since $c \neq 0$, the system (6.8) can be rewritten in the following form

$$
\begin{aligned}
\boldsymbol{A}_{N I}\left(\begin{array}{c}
\psi_{0} \\
v_{0}
\end{array}\right)=\left(\begin{array}{c}
G \\
K
\end{array}\right), \quad \boldsymbol{A}_{N I}:=\boldsymbol{A}_{N I}^{0}+T, \\
\boldsymbol{A}_{N I}^{0}:=\left(\begin{array}{cc}
\frac{1}{c} \boldsymbol{V}_{\mathbb{R},+1} & -\frac{1}{c} \boldsymbol{V}_{\mathbb{R},+1} \\
\frac{1}{2} I & 0
\end{array}\right) \\
\quad \begin{array}{lll}
\mathbb{\mathbb { W }}_{p}^{s-1-1 / p}\left(\mathbb{R}^{+}\right) \\
\mathbb{\mathbb { W }}_{p}^{s-1-1 / p}\left(\mathbb{R}^{-}\right)
\end{array} \rightarrow \mathbb{W}_{p}^{s-2-1 / p}(\mathbb{R}) \\
\mathbb{W}_{p}^{s-1-1 / p}(\mathbb{R}),
\end{aligned}
$$

where the first and second rows of the operator

$$
\boldsymbol{T}:=\left(\begin{array}{cc}
\frac{1}{2} I & 0 \\
-c \boldsymbol{V}_{\mathbb{R},-1} & -\frac{1}{2} I
\end{array}\right): \begin{aligned}
& \widetilde{\mathbb{W}}_{p}^{s-1-1 / p}\left(\mathbb{R}^{+}\right) \\
& \widetilde{\mathbb{W}}_{p}^{s-1-1 / p}\left(\mathbb{R}^{-}\right)
\end{aligned} \rightarrow \begin{aligned}
& \mathbb{W}_{p}^{s-2-1 / p}(\mathbb{R}) \\
& \mathbb{W}_{p}^{s-1-1 / p}(\mathbb{R})
\end{aligned}
$$

have orders 0 and -1 , respectively and, therefore, $\boldsymbol{T}$ is locally compact (recall that here $I$ is a compact operator due to the Rellich-Kondrachov compact embedding theorem). Then $\boldsymbol{T}$ is locally equivalent to 0 at 0 .

On the other hand the operator $\boldsymbol{V}_{\mathbb{R},+1}$ is Fredholm: it has non-trivial kernel but there exists regularizer $\boldsymbol{R}_{-1}$ such that

$$
\boldsymbol{R}_{-1} \boldsymbol{V}_{\mathbb{R},+1}=I+\boldsymbol{T}_{1}, \quad \boldsymbol{V}_{\mathbb{R},+1} \boldsymbol{R}_{-1}=I+\boldsymbol{T}_{2},
$$


where $\boldsymbol{T}_{1}$ is a compact operator in $\widetilde{\mathbb{W}}_{p}^{s-1-1 / p}\left(\mathbb{R}^{+}\right)$while $\boldsymbol{T}_{2}$ is compact in $\mathbb{W}_{p}^{s-2-1 / p}\left(\mathbb{R}^{+}\right)$. Since $\boldsymbol{T}_{1}$ and $\boldsymbol{T}_{2}$ are locally equivalent to 0 at 0 , the regularizer $\boldsymbol{R}_{-1}$ turns out to be a local inverse to $\boldsymbol{V}_{\mathbb{R},+1}$ at 0 . Therefore the local inverse to $\boldsymbol{A}_{N I}$ at 0 is the operator $\left(\begin{array}{cc}0 & 2 I \\ -c \boldsymbol{R}_{-1} & 2 I\end{array}\right)$.

Remark 2. In all the three cases, the operator associated to the BVP is equivalent after extension to the operator associated to the corresponding semi-homogeneous problem, and that one is equivalent to the operator associated to the corresponding system of boundary pseudodifferential equations. The transitivity of equivalent after extension relations and of the local equivalence relations give the full result, in combination of [11] and [44].

7. Proofs of the main theorems. We are now in a position to prove Theorem 2 and Theorem 1, based on the following auxiliary proposition.

Proposition $7([\mathbf{1 6}, \mathbf{2 0}, \mathbf{2 1}])$. Let two pairs of parameter-dependent Banach spaces $\mathfrak{B}_{1}^{s}$ and $\mathfrak{B}_{2}^{s}, s_{1}<s<s_{2}$, have intersections $\mathfrak{B}_{j}^{s^{\prime}} \cap \mathfrak{B}_{j}^{s^{\prime \prime}}$ dense in $\mathfrak{B}_{j}^{s^{\prime}}$ and in $\mathfrak{B}_{j}^{s^{\prime \prime}}$ for all $j=1,2, s^{\prime}, s^{\prime \prime} \in\left(s_{1}, s_{2}\right)$.

If a linear bounded operator $A: \mathfrak{B}_{1}^{s} \rightarrow \mathfrak{B}_{2}^{s}$ is Fredholm for all $s \in\left(s_{1}, s_{2}\right)$, it has the same kernel and cokernel for all values of this parameter $s \in\left(s_{1}, s_{2}\right)$.

In particular, if $A: \mathfrak{B}_{1}^{s} \rightarrow \mathfrak{B}_{2}^{s}$ is Fredholm for all $s \in\left(s_{1}, s_{2}\right)$ and is invertible for one value $s_{0} \in\left(s_{1}, s_{2}\right)$, then it is invertible for all values of this parameter $s \in\left(s_{1}, s_{2}\right)$.

Consider the following equation on the two-dimensional Euclidean space

$$
\Delta u=f^{0} \quad \text { on } \quad \mathbb{R}^{2}, \quad u \in \mathbb{H}_{p}^{s}\left(\mathbb{R}^{2}\right), \quad f^{0} \in \mathbb{H}_{p}^{s-2}\left(\mathbb{R}^{2}\right),
$$

and the model boundary value problems for the Laplace equation on 
the upper half-plane $\mathbb{R}_{+}^{2}:=\mathbb{R} \times \mathbb{R}^{+}$: the model Dirichlet

$$
\begin{cases}\Delta u(x)=f_{0}(x), & x \in \mathbb{R}_{+}^{2}, \\ u^{+}(t)=g_{0}(t), & t \in \partial \mathbb{R}_{+}^{2}=\mathbb{R},\end{cases}
$$

the model Neumann

$$
\begin{cases}\Delta u(x)=f_{0}(x), & x \in \mathbb{R}_{+}^{2}, \\ -\left(\partial_{2} u\right)^{+}(t)=h_{0}(t), & t \in \partial \mathbb{R}_{+}^{2}=\mathbb{R}\end{cases}
$$

and the model impedance BVP

$$
\begin{cases}\Delta u(x)=f_{0}(x), & x \in \mathbb{R}_{+}^{2}, \\ -\left(\partial_{2} u\right)^{+}(t)+c u^{+}(t)=\kappa_{0}(t), & t \in \partial \mathbb{R}_{+}^{2}=\mathbb{R},\end{cases}
$$

The BVPs (7.1)-(7.4) will be treated in the nonclassical setting:

$$
\begin{gathered}
f_{0} \in \widetilde{\mathbb{H}}_{p}^{s-2}\left(\mathbb{R}_{+}^{2}\right) \cap \widetilde{\mathbb{H}}_{0}^{-1}\left(\mathbb{R}_{+}^{2}\right), \quad g_{0} \in \mathbb{W}_{p}^{s-1 / p}(\mathbb{R}), \\
h_{0}, \kappa_{0} \in \mathbb{W}_{p}^{s-1-1 / p}(\mathbb{R}), \quad 1<p<\infty, \quad s>\frac{1}{p} .
\end{gathered}
$$

Proposition 8. The Laplace equation (7.1) and the BVPs (7.2)-(7.4) have unique solutions in the setting (7.5).

Proof: For $p=2, s=1$, the assertion is a well-known classical result, available in many books on partial differential equations (see, e.g., [27]). It can be proved similarly to Theorem 3 by applying the Lax-Milgram Lemma 1.

Now let $p \neq 2$. Note that the BVPs (7.2)-(7.4) and the corresponding boundary integral equations (BIEs) are equivalent (their solutions are related one-to-one). The BIEs on $\mathbb{R}$ have unique solutions for $p=2$, $s=1$ and are Fredholm in the spaces $\mathbb{H}_{p}^{s}(\mathbb{R})$ for all $-\infty<s<\infty$, $1<p<\infty$. Therefore, due to Proposition 7 BIEs have unique solutions in the spaces $\mathbb{H}_{p}^{s}(\mathbb{R})$ for all $-\infty<s<\infty, 1<p<\infty$, which means that the corresponding BVPs (7.2)-(7.4) also have unique solutions in the setting (7.5).

Proof of Theorem 2: The MIND BVP (1.2) is Fredholm in the nonclassical setting (3.3) if and only if all three model mixed BVPs 
Dirichlet-Neumann (5.1), Dirichlet-impedance (5.2) and Neumannimpedance (5.3) are locally invertible at 0 in the nonclassical setting (5.4)-(5.5) (cf. Lemma 4). Due to Theorems 4-6 this is the case if and only if the condition (3.5) holds.

Now let $c>0$. Due to the proved part of the present theorem the MIND BVP (1.2) is Fredholm if the point $(s, 1 / p)$ belongs to the open curved quadrangle $A B C D$ in Figure 1 . On the other hand, for the particular space parameters $p=2, s=1$, which is the center of the open curved quadrangle $A B C D$ in Figure 1, the MIND BVP (1.2) has a unique solution (see Theorem 3). Then, due to Proposition 7, the MIND BVP (1.2) has a unique solution in the nonclassical setting (3.3) for all those $s$ and $p$ for which the point $(s, 1 / p)$ belong to the open curved quadrangle $A B C D$ in Figure 1.

Proof of Theorem 1: Since the point $(1,1 / 2)$ (i.e., $s=1, p=2$ ) is the center of the quadrangle $A B C D$ in Figure 1, due to Theorem 2 the corresponding BVP has a unique solution for all real and complex values of the nonzero parameter $c \in \mathbb{C}$.

Remark 3. One may ask: why we did not derive also Theorem 3 from Theorem 2? The answer is simple: we apply Theorem 3 (but not Theorem 1) in the proof of Theorem 2 (see the proof of Theorem 2 above).

Acknowledgements. Research supported in part by the grant of the Shota Rustaveli Georgian National Science Foundation GNSF/DI2016-16, and by FCT-Portuguese Foundation for Science and Technology through the CIDMA-Center for Research and Development in Mathematics and Applications at Universidade de Aveiro, within the project UIDB/04106/2020, and the CEAFEL-Center for Functional Analysis, Linear Structures and Applications at IST, Universidade de Lisboa.

\section{REFERENCES}

1. A.-S. Bonnet-Ben Dhia, L. Chesnel, P. Ciarlet Jr., T-coercivity for scalar interface problems between dielectrics and metamaterials, ESAIM: Mathematical Modelling and Numerical Analysis 46, 2012, 1363-1387. 
2. A.-S. Bonnet-Ben Dhia, A. Tillequin, A limiting absorption principle for scattering problems with unbounded obstacles, Math. Meth. Appl. Sci. 24, 2001, 1089-1111.

3. T. Buchukuri, O. Chkadua, R. Duduchava, D. Natroshvili, Interface crack problems for metallic-piezoelectric, Mem. Differ. Equ. Math. Phys. 55, 2012, 1150.

4. L.P. Castro, D. Kapanadze, On wave diffraction by a half-plane with different face impedances, Math. Methods Appl. Sci. 30, 2007, 513-527.

5. L.P. Castro, D. Kapanadze, Dirichlet-Neumann-impedance boundary value problems arising in rectangular wedge diffraction problems, Proc. Am. Math. Soc. 136, 2008, 2113-2123.

6. L.P. Castro, D. Kapanadze, The impedance boundary-value problem of diffraction by a strip, J. Math. Anal. Appl. 337, 2008, 1031-1040.

7. L.P. Castro, D. Kapanadze, Wave diffraction by a strip with first and second kind boundary conditions: The real wave number case, Math. Nachr. 281, 2008, 1400-1411.

8. L.P. Castro, D. Kapanadze, Exterior wedge diffraction problems with Dirichlet, Neumann and impedance boundary conditions, Acta Applicandae Mathematicae 110, 2010, 289-311.

9. L.P. Castro, D. Kapanadze, Wave diffraction by a half-plane with an obstacle perpendicular to the boundary, J. Differ. Equations 254, 2013, 493-510.

10. L.P. Castro, D. Kapanadze, Wave diffraction by wedges having arbitrary aperture angle, J. Math. Anal. Appl. 421, 2015, 1295-1314.

11. L.P. Castro, R. Duduchava, F.-O. Speck, Localization and minimal normalization of some basic mixed boundary value problems. Factorization, Singular Operators and Related Problems, Proceedings of the Conference in Honour of Professor Georgii Litvinchuk, Portugal 2002 (Eds. S. Samko et al.), Kluwer, Dordrecht, 2003, 73-100.

12. L.P. Castro, F.-O. Speck, F.S. Teixeira, Explicit solution of a DirichletNeumann wedge diffraction problem with a strip, J. Integral Equations Appl. 15, 2003, 359-383.

13. L.P. Castro, F.-O. Speck, F.S. Teixeira, On a class of wedge diffraction problems posted by Erhard Meister, Oper. Theory Adv. Appl. 147, 2004, 211-238.

14. L.P. Castro, F.-O. Speck, F.S. Teixeira, Mixed boundary value problems for the Helmholtz equation in a quadrant, Integral Equations and Operator Theory $\mathbf{5 6}$, 2006, 1-44.

15. V. Didenko, R. Duduchava, Mellin convolution operators in Bessel potential spaces with admissible meromorphic kernels, Journal of Analysis and Applications 443, 2016, 707-731.

16. R. Duduchava, On Noether theorems for singular integral equations, Proceedings of Symposium on Mechanics and Related Problems of Analysis 1, Metsniereba, Tbilisi, 1973, 19-52 (in Russian). 
17. R. Duduchava, The Green formula and layer potentials, Integral Equations and Operator Theory 41, 2001, 127-178.

18. R. Duduchava, Partial differential equations on hypersurfaces, Mem. Differ. Equ. Math. Phys. 48, 2009, 19-74.

19. R. Duduchava, M. Tsaava, Mixed boundary value problems for the LaplaceBeltrami equation, Complex Variables and Differential Equations 63, 10, 2018, 1468-1496.

20. R. Duduchava, D. Natroshvili, E. Shargorodsky, Basic boundary value problems of thermoelasticity for anisotropic bodies with cuts I, Georgian Mathematical Journal 2, 1995, 123-140.

21. R. Duduchava, D. Natroshvili, E. Shargorodsky, Basic boundary value problems of thermoelasticity for anisotropic bodies with cuts II, Georgian Mathematical Journal 2, 1995, 259-276.

22. R. Duduchava, D. Mitrea, M. Mitrea, Differential operators and boundary value problems on surfaces, Mathematische Nachrichten 279, 2006, 996-1023.

23. R. Duduchava, M. Tsaava, T. Tsutsunava, Mixed boundary value problem on hypersurfaces, International Journal of Differential Equations 2014, Article ID 245350, 8 pp.

24. T. Ehrhardt, A.P. Nolasco, F.-O. Speck, Boundary integral methods for wedge diffraction problems: the angle $2 \pi / n$, Dirichlet and Neumann conditions, Operators and Matrices 5, 2011, 1-40.

25. T. Erhardt, A.P. Nolasco, F.-O. Speck, A Riemannn surface approach for diffraction from rational wedges, Operators and Matrices 8, 2014, 301-355.

26. I. Gohberg, N. Krupnik, One-Dimensional Linear Singular Integral Equations I-II, Oper. Theory Adv. Appl. 53-54, Birkhäuser, Basel, 1979.

27. G.C. Hsiao, W.L. Wendland, Boundary Integral Equations, Applied Mathematical Sciences, Springer-Verlag, Berlin, 2008.

28. D. Kapanadze, B.-W. Schulze, Crack Theory and Edge Singularities, Kluwer Academic Publishers, Dordrecht, 2003.

29. A.I. Komech, N.J. Mauser, A.E. Merzon, On Sommerfeld representation and uniqueness in scattering by wedges, Math. Meth. Appl. Sci. 28, 2005, 147-183.

30. V.A. Kozlov, V.G. Maz'ya, J. Rossmann, Spectral Problems Associated with Corner Singularities of Solutions to Elliptic Equations, AMS, Providence, 2001.

31. P.D. Lax, A.N. Milgram, Parabolic equations. Contributions to the theory of partial differential equations, pp. 167-190. Annals of Mathematics Studies 33. Princeton University Press, Princeton, N.J., 1954.

32. J.L. Lions, E. Magenes, Non-homogeneous Boundary Value Problems and Applications I, Springer-Verlag, Heidelberg, 1972.

33. G.D. Malyuzhinets, The excitation, reflection and radiation of surface waves in a wedge-like region with given face impedances, Dokl. Acad. Nauk SSSR 121, 1958, 436-439.

34. E. Meister, Some multiple-part Wiener-Hopf problems in Mathematical 
Physics, Mathematical Models and Methods in Mechanics, Banach Center Publications 15, PWN - Polish Scientific Publishers, Warsaw, 1985, 359-407.

35. E. Meister, Some solved and unsolved canonical problems of diffraction theory, Lecture Notes in Math. 1285, 1987, 320-336.

36. E. Meister, F.-O. Speck, Some multidimensional Wiener-Hopf equations with applications, Trends in Applications of Pure Mathematics to Mechanics 2, Pitman, London, 1979, 217-262.

37. E. Meister, F.-O. Speck, Modern Wiener-Hopf methods in diffraction theory, Ordinary and Partial Differential Equations II, Proc. 10th Dundee Conf., Dundee/UK 1988, Pitman Res. Notes Math. Ser. 216, 1989, 130-171.

38. E. Meister, F. Penzel, F.-O. Speck, F.S. Teixeira, Some interior and exterior boundary value problems for the Helmholtz equation in a quadrant, Proc. $R$. Soc. Edinb., Sect. A, 123, 1993, 193-237.

39. A. Moura Santos, F.-O. Speck, F.S. Teixeira, Minimal normalization of Wiener-Hopf operators in spaces of Bessel potentials, J. Math. Anal. Appl. 225, 1998, 501-531.

40. B. Noble, Methods Based on the Wiener-Hopf Technique, Pergamon, London, 1958.

41. A.F. dos Santos, F.S. Teixeira, The Sommerfeld problem revisited: solution spaces and the edge conditions, J. Math. Anal. Appl. 143, 1989, 341-357.

42. A.M. Santos, F.-O. Speck, F.S. Teixeira, Compatibility conditions in some diffraction problems, Direct and inverse electromagnetic scattering, Pitman Res. Notes Math. Ser. 361, 1996, 25-38.

43. F.-O. Speck, Mixed boundary value problems of the type of Sommerfeld halfplane problem, Proc. Royal Soc. Edinburgh 104 A, 1986, 261-277.

44. F.-O. Speck, On the reduction of linear systems related to boundary value problems, Oper. Theory Adv. Appl. 228, 2013, 391-406.

45. F.-O. Speck, R.A. Hurd, E. Meister, Sommerfeld diffraction problems with third kind boundary conditions, SIAM J. Math. Anal. 20, 1989, 589-607.

46. H. Triebel, Interpolation Theory, Function Spaces, Differential Operators (2nd edt.), Johann Ambrosius Barth Verlag, Heidelberg, 1995.

47. P.Ya. Ufimtsev, Theory of Edge Diffraction in Electromagnetics, Tech Science Press, Encino, California, 2003.

48. V.B. Vasil'ev, Wave Factorization of Elliptic Symbols: Theory and Applications, Kluwer Academic Publishers, Dordrecht, 2000.

49. W.L. Wendland, E. Stephan, G.C. Hsiao, On the integral equation method for the plane mixed boundary value problem of the Laplacian, Math. Methods Appl. Sci. 1, 1979, 265-321.

50. P. Zhevandrov, A. Merzon, On the Neumann problem for the Helmholtz equation in a plane angle, Math. Meth. Appl. Sci. 23, 2000, 1401-1446. 
Cidma - Center for Research and Development in Mathematics and Applications, Department of Mathematics, University of Aveiro, Portugal Email address: castro@ua.pt

The University of Georgia \& A. Razmadze Mathematical Institute, Tbilisi, Georgia

Email address: R.Duduchava@ug.edu.ge

CeAfel - Center for Functional Analysis, Linear Structures and AppliCATions, IST, University of Lisbon, Portugal

Email address: fspeck@tecnico.pt 